\title{
Analysis of Digital Image Filters in Frequency Domain
}

\author{
Md. Shahnawaz Shaikh \\ Assistant Professor \\ Department of ECE \\ SDITS Khandwa
}

\author{
Ankita Choudhry \\ Final year Student \\ Department of ECE \\ SDITS Khandwa
}

\author{
Rakhi Wadhwani \\ Final year Student Department \\ of ECE \\ SDITS Khandwa
}

\begin{abstract}
Digital image filtering in an important approach towards image enhancement. It involves the manipulation and interpretation of digital images. Images may get noisy due to various factors then filtering of images is become an important operation to de-noise the noisy images. Image Smoothing and image sharpening must be performed to achieve it. In our paper we work on Ideal, Gaussian and Butterworth filters to apply high pass and low pass filtering on images. We implement and simulate these filters on MATLAB platform and analyze their performance for equal cutoff frequency. This work gives great experience to understand differences among various above define filtering techniques through keen analysis of their respective results.
\end{abstract}

\section{Keywords}

Frequency domain image filtering, high pass filter, low pass filter, Ideal filter, Butterworth filter, Gaussian filter..

\section{INTRODUCTION}

Frequency domain filtering of digital images involves conversion of digital images from spatial domain to frequency domain. Frequency domain image filtering is the process of image enhancement other than enhancement in spatial domain, which is an approach to enhance images for specific application. In this paper we discussed about frequency domain approach of image filtering. As normally images are in spatial domain, so it needed its conversion in frequency domain. Fast Fourier transform is a specific tool for spatial domain to frequency domain conversion. For image smoothing we implement low pass filters while for image sharpening we implement high pass filters. Both low pass and high pass filtering implemented and analyze for Ideal filter, Butterworth filter and Gaussian filter. Filtering in frequency domain is based on modifying the Fourier transform of image of interest to achieve frequency domain filtering and then get back spatial domain image by taking inverse Fourier transform of resultant filtered image [1-2], [4], [5-8], [10].

The first section of this paper introduces to our work. Frequency domain with basics of frequency domain conversion enlightened in section two. This section also contain algorithm to perform frequency domain filtering. Section three elaborates types of frequency domain filters which we implemented and analyze. Analysis of all implementations and comparisons of their performance using resulting images discussed in section four. Section five concluded our work and future scopes which could be done.

\section{FREQUENCY DOMAIN FILTERING}

Frequency domain is basically a space defined by Fourier transform. Fourier transforms has wide applications in image processing, such as image analysis, image filtering, image reconstruction and image compression [9]. In frequency domain analysis, it indicates that how signal energy is distributed over a range of frequencies. The basic principle of the frequency domain image filtering consists of computing a 2-D discrete Fourier transform of the image, for instance the 2-D DFT, manipulating the transform coefficients by an operator i.e. filter function and then performing the inverse discrete Fourier transform [4], [8].

\subsection{Steps for filtering in the frequency domain}

1. Let original image $f(x, y)$ of size $M X N$ that is discrete image.

2. Apply zero padding to $f(x, y)$ to get image $f p(x, y)$ of size $P X Q$. such that $P=2 M$ and $Q=2 N$. Padding means appending necessary number of zeros to original image $f(x, y)$.

3. Multiply the padded image $f p(x, y)$ by $(-1)^{x+y}$ to center the transform to $u=P / 2$ And $v=Q / 2$. Where $u$ and $v$ are center coordinate of padded image.

4. Compute the 2D-DFT of centered image $f(u, v)$.

5. Define filter function $h(x, y)$ of size $P X Q$ get $H(u, v)$ that is FFT of $h(x, y)$

6. Centralize the coordinate of $H(u, v)$ at $(P / 2, Q / 2)$.

7. Multiply $F(u, v)$ by a filter function $H(u, v)$.

8. Compute the inverse DFT of the result.

9. Crop the result in 6 .

10. Obtain the real part of the result in 6 .

11. Multiply the result in (6) by $(-1)^{x+y}$ to reverse centering of the output image as input image [1], [8]. 


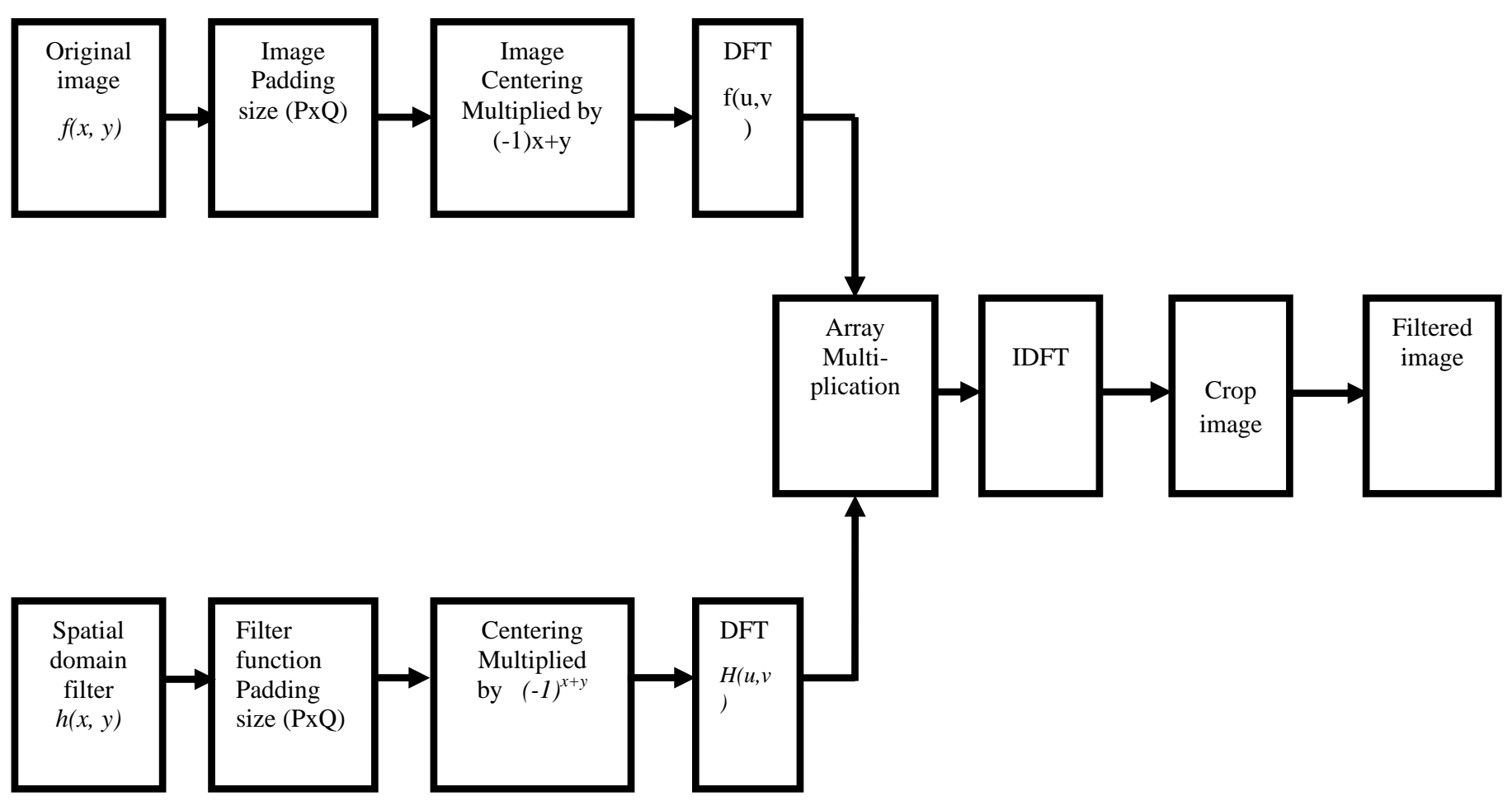

Fig 2.1: Frequency domain filtering process [1].

Figure 1 shows the whole process involve in frequency domain image filtering. Fourier transform converts time domain to frequency domain while inverse Fourier transforms converts frequency domain back to time domain function. Intensity of image depends on frequency domain of Fourier transform.

\subsection{Discrete Fourier Transforms}

2-D Fast Fourier transform (2D- DFT) given as

$$
f(u, v)=\sum_{X=0}^{M-1} \sum_{Y=0}^{N-1} f(x, y) e^{-j 2 \pi\left(\frac{u x}{M}+\frac{v y}{N}\right)}
$$

Equation .2.1

In equation $2.1, f(x, y)$ is the spatial domain $2 \mathrm{D}$ - function, which representing spatial domain image while $f(u, v)$ represents its frequency domain representation [1], [2], [5], [8].

If we consider $u=v=0$, means slowest varying frequency component.

$f(0,0)=\sum_{N-1}^{M-1} \sum_{N-1}^{M-1} f(x, y)$

Equation .2 .2

In equation 2.2 indicates zero frequency term of $f(u, v)$. Low frequencies indicate slow variation of intensity, while as we move further away from the origin of transform, high frequency component indicates that fast variation of intensity.

\subsection{Inverse Discrete Fourier Transforms}

The inverse discrete Fourier transform (IDFT) is used to recover the spatial domain image from frequency domain image. The equation The IDFT of $f(u, v)$ is given by [1], [2], [5].

$$
f(x, y)=\frac{1}{M N} \sum_{u=0}^{M-1} \sum_{v=0}^{N-1} f(u, v) e^{j 2 \pi\left(\frac{u x}{M}+\frac{v y}{N}\right)} \ldots 2.3 .1
$$

For $\mathrm{x}=0,1,2 \ldots \mathrm{M}-1$ and $\mathrm{y}=0,1,2 \ldots \mathrm{N}-1 \ldots$ Equation .2 .3

\section{FREQUENCY DOMAIN FILTERS}

\subsection{Image enhancement}

Image Enhancement improves the perception of information in images for human viewers. The deteriorated image due to optics, electronics or environment may be enhanced to restore certain features of an image. The image may corrupted by different types of noise such as additive noise, Gaussian

noise, impulse noise and Poisson noise etc. To remove these types of noises there are various filters are available [4], [7].

The basic operations performed with noisy image is smoothing and sharpening just as low pass filtering and high pass filtering. Smoothing or blurring of noisy images is analogous to the low pass filtering while sharpening of images represent high pass filtering [1], [8].

The term frequency in images has very interesting definition. It is just representing the changes or transition in pixel intensities. Low intensity transition from pixel to pixel shows low frequency however high frequency indicates high intensity transition from pixel to pixel. The term cutoff frequency in image filter represented by the pixel distance from center pixel of the image, usually it is denoted by $D_{0}[1]$, [8].

\subsection{Low pass filter}

Low pass filter perform smoothing or blurring of images. It is achieved in frequency domain by attenuation of high frequency or high intensity transition. The output of low pass filter contains small or less intensity transition in to a particular group of pixels. We implement Ideal low pass filter 
(ILPF), Butterworth low pass filter (BLPF) and Gaussian low pass filter (GLPF) on MATLAB platform and analyze it

\subsubsection{Ideal low pass filter (ILPF)}

ILPF gives ideal response for image filtering. It passes all the frequency within a circle of radius $D_{0}$ which is cutoff frequency of ILPF, while attenuates all the frequency lies outside this circle. It is specified by the function in equation 3.1 .

$$
H(u, v)= \begin{cases}1 & \text { if } \mathrm{D}(\mathrm{u}, \mathrm{v}) \leq \mathrm{D} 0 \\ 0 & \text { if } \mathrm{D}(\mathrm{u}, \mathrm{v})>D 0\end{cases}
$$

Equation .3 .1

Where $D(u, v)$ is the distance between center of the frequency rectangle and a point $(u, v)$ in the frequency domain image calculated by equation 3.2 [2], [5], [8], [9].

$$
D(u, v)=\sqrt{\left(u-\frac{p}{2}\right)^{2}+\left(v-\frac{Q}{2}\right)^{2}}
$$

Equation .3 .2

$\left[\frac{P}{2}, \frac{Q}{2}\right]$ Defines the center of circle of radius $D_{0}$.

\subsubsection{Butterworth low pass filter (BLPF)}

Butterworth filter is very popular filter due to its varying performance depends on its order. An Increased ordered Butterworth filter tends its performance towards ideal filter.The filter function of BLPF is given by equation 3.3

$$
H(u, v)=\frac{1}{1+\left[\frac{D(u, v)}{D 0}\right]^{2 n}}
$$

Equation .3 .3

Where $n$ is the filter order. $D(u, v)$ and $D_{0}$ has same meaning as defined above [2], [5], [8], [9].

\subsubsection{Gaussian low pass filter (GLPF)}

Gaussian filter is the generalized filter for digital images. Filter function for GLPF is denoted by equation 3.4

$$
H(u, v)=e^{\frac{-D^{2}(u, v)}{2 D 0^{2}}}
$$

Equation .3 .4

Where $D(u, v)$ and $D_{0}$ has same meaning as defined above [2], [5], [8], [9].

\subsection{High pass filter}

High pass filter performs sharpening of images. It is achieved in frequency domain by attenuation of low frequency or low intensity transition. The output of high pass filter contains high intensity transition in to a particular group of pixels. We implement Ideal high pass filter (IHPF), Butterworth high pass filter (BHPF) and Gaussian high pass filter (GHPF) on MATLAB platform and analyze it.

\subsubsection{Ideal high pass filter (IHPF)}

The filter function of IHPF is given by equation 3.5

$$
H(u, v)= \begin{cases}0 & \text { if } \mathrm{D}(\mathrm{u}, \mathrm{v}) \leq \mathrm{D} 0 \\ 1 & \text { if } \mathrm{D}(\mathrm{u}, \mathrm{v})>D 0\end{cases}
$$

Equation .3 .5

It passes all the frequency outside the circle of radius $D_{0}$ which is cutoff frequency of IHPF, while attenuates all the frequency lies within this circle. $D(u, v)$ and $D_{0}$ has same meaning as defined above [2], [5], [8], [9].

\subsubsection{Butterworth high pass filter (BLPF)}

The filter function of BHPF is given by equation 3.6

$$
H(u, v)=\frac{1}{1+\left[\frac{D 0}{D(u, v)}\right]^{2 n}}
$$

Equation .3.6

Where $D(u, v)$ and $D_{0}$ has same meaning as defined above and $n$ is the order of filter [2], [5], [8], [9].

\subsubsection{Gaussian high pass filter (GHPF)}

Filter function for GLPF is denoted by equation 3.7 [2], [5], [9].

$$
H(u, v)=1-e^{\frac{-D^{2}(u, v)}{2 D 0^{2}}}
$$

Equation .3 .7

\section{RESULTS AND ANALYSIS}

Simulation performed for Ideal, Butterworth, and Gaussian filters for both the low pass filtering and high pass filtering on MATLAB platform. Analysis of these results gives interesting conclusions. All simulation done for same image of resolution $1024 \times 768$ pixels grayscale image. Cutoff frequency taken for low pass filtering $D_{0}=200$, also for high pass filtering it is taken $D_{0}=200$. All the Ideal, Butterworth, and Gaussian filters are simulated for equal cutoff frequencies either for low pass filtering or high pass filtering. 


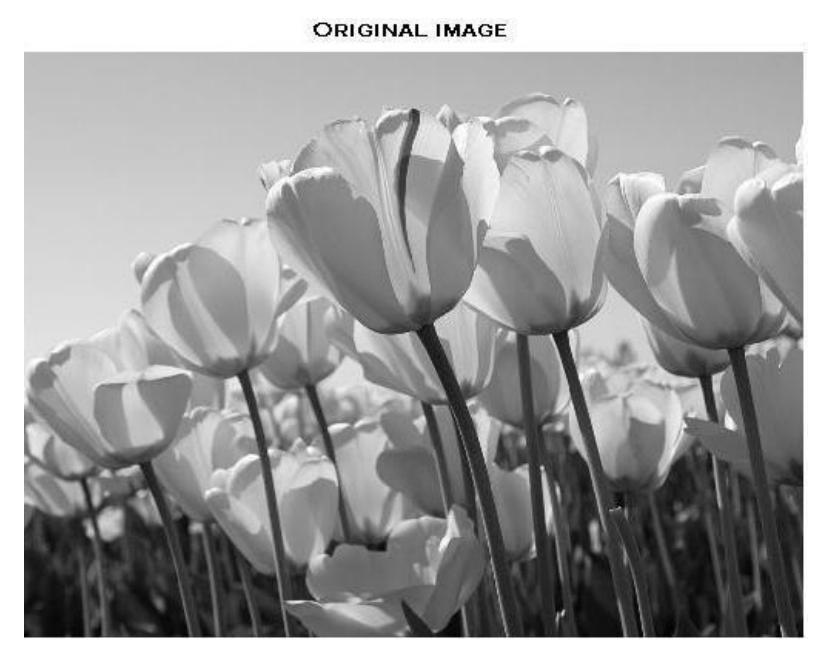

Figure 4.1: Original grayscale image

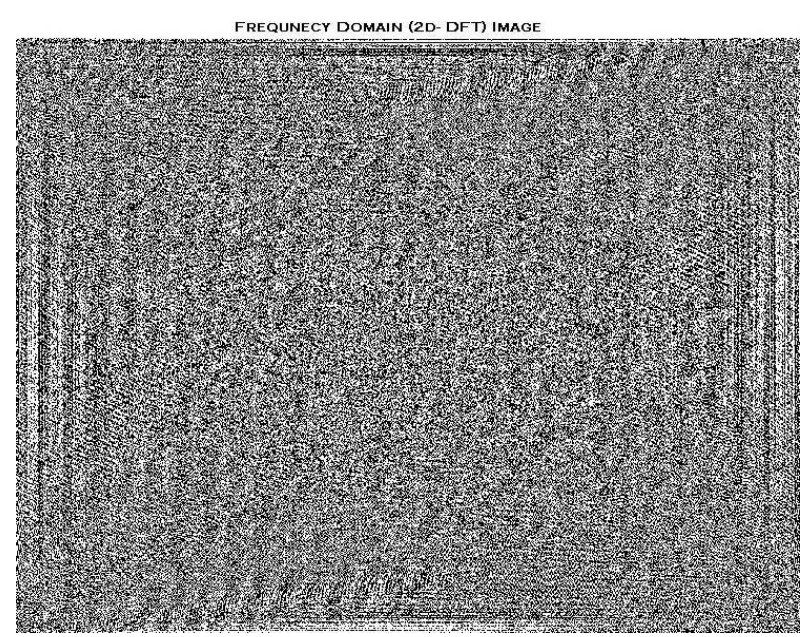

Figure 4.2: Frequency domain (2D- FFT) image

Figure 4.1 shows the image of interest at which operation is performed. Figure 4.2 is the frequency domain view while taking 2D-DFT of image shown in figure 4.1

\subsection{Ideal low pass filter}

Figure 4.3 shows ideal filter of cutoff frequency $D_{0}=200$. Ideal filter shows ideal characteristics i.e. its circle boundary is sharp which results high degree of smoothing of image of interest. Figure 4.4 shows filtered image through Ideal low pass filter i.e. smoothed image of input gray scale image.

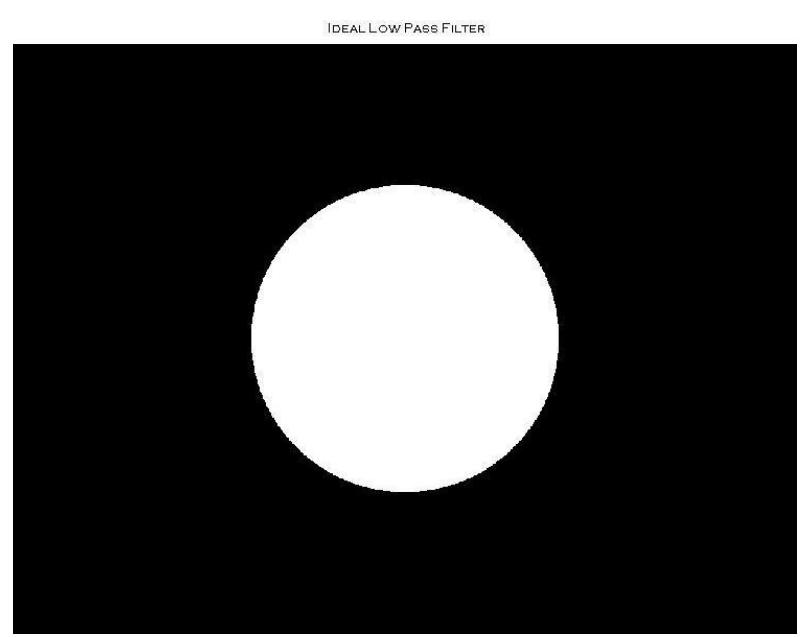

Figure 4.3: Ideal low pass filter (ILPF) of cutoff frequency $200, D_{0}=200$

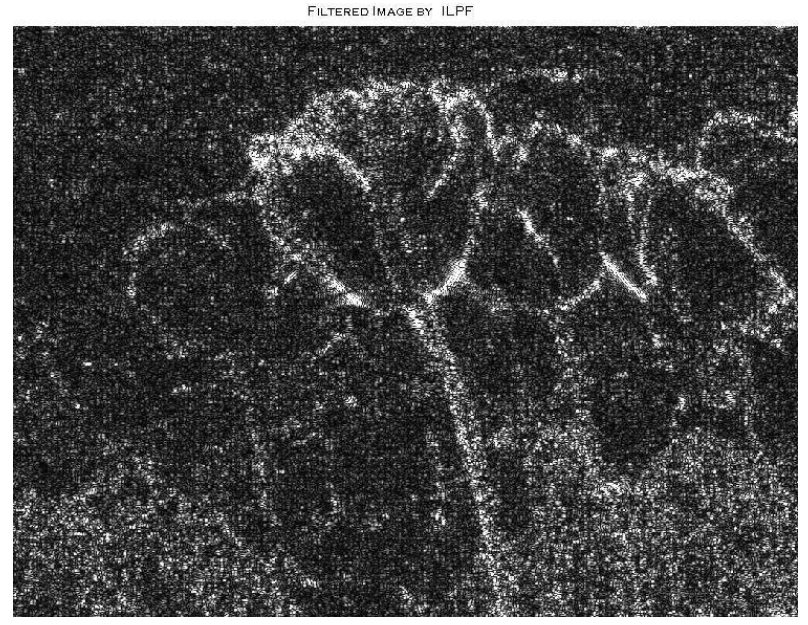

Figure 4.4: ILPF filtered image

\subsection{Butterworth low pass filter}

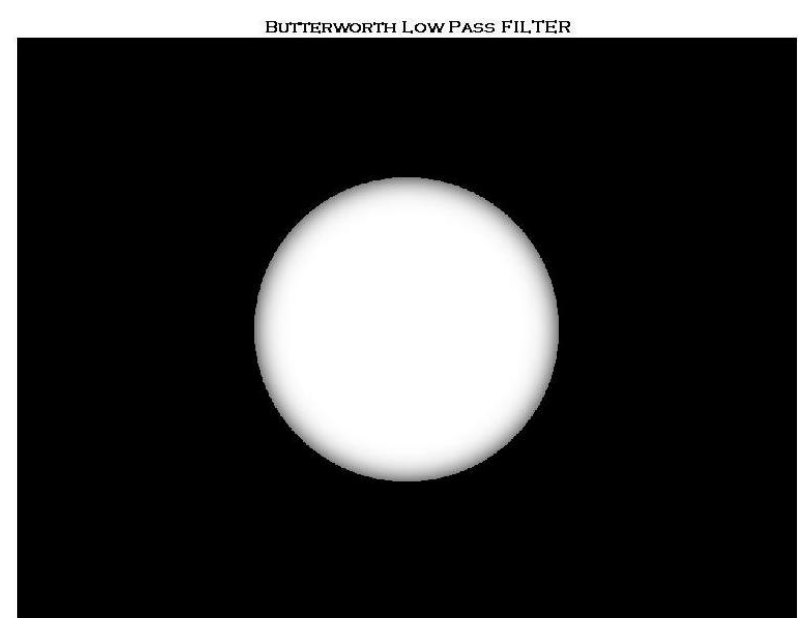

Figure 4.5 : Butterworth low pass filter (BLPF) of cutoff frequency $200, D_{0}=200$, order $n=10$ 


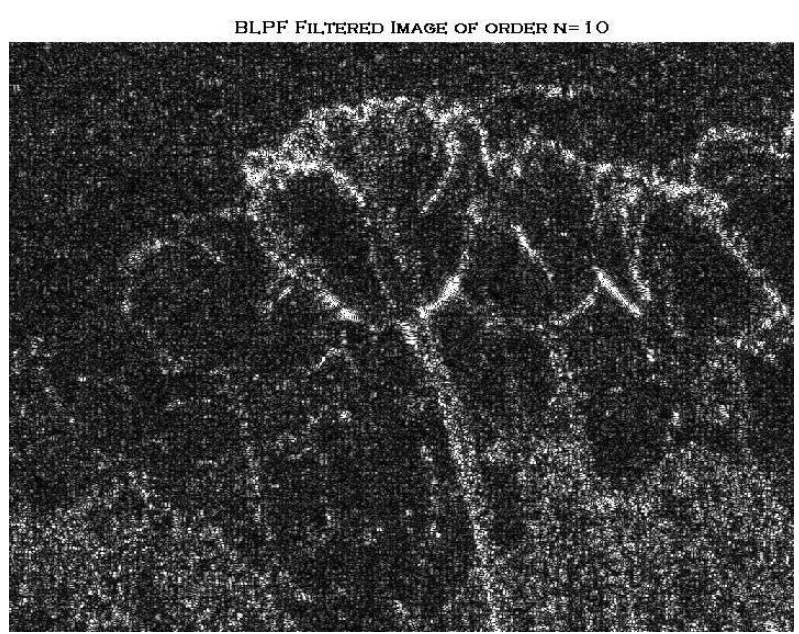

Figure 4.6 : BLPF filtered image of cutoff frequency 200, $D_{0}=200$, order $n=10$

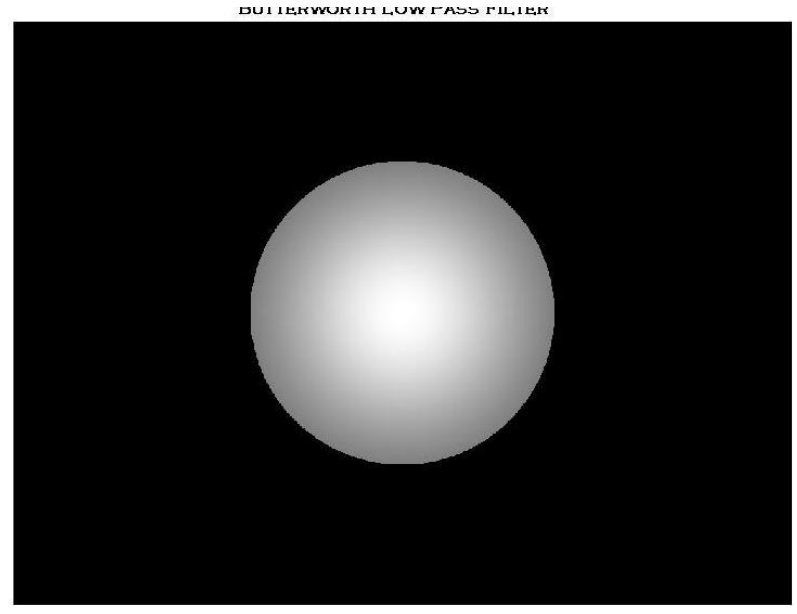

Figure 4.7 : Butterworth low pass filter (BLPF) of cutoff frequency $200, D_{0}=200$, order,$n=1$

BLPF FILTERED IMAGE WTH ORDER $\mathbf{N}=1$

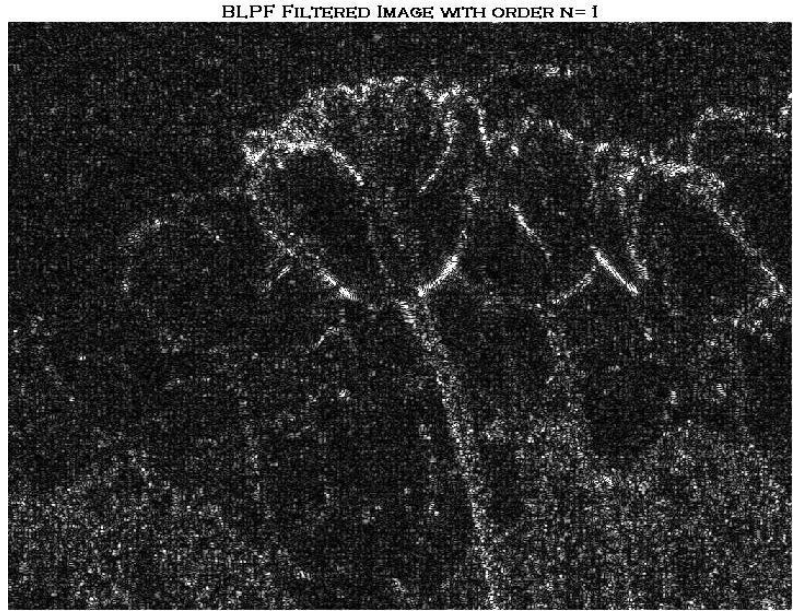

Figure 4.8: BLPF filtered image of cutoff frequency 200, $D_{0}=200$, order, $n=1$

Figure 4.5 shows Butterworth low pass tilter of cutotf frequency $D_{0}=200$ and order $n=10$. While figure 4.7 is the same filter with reduced order i.e. $n=1$. Its circle boundary is not sharp as ideal filter. It is somewhat blurred at its boundary. It can also be analyzed that as the order of Butterworth filter increases, its circle boundary is getting sharp. We can say increasing the Butterworth filter order is approaching towards ideal filter characteristics.

Figure 4.6 shows filtered output image through filter shown in figure 4.5 i.e. higher order filter, while figure 4.8 shows filtered output image through filter shown in figure 4.7 i.e. lower order filter. Higher order Butterworth low pass filter gives better smoothing result than lower order filter. It is almost near to ILPF output.

\subsection{Gaussian low pass filter}

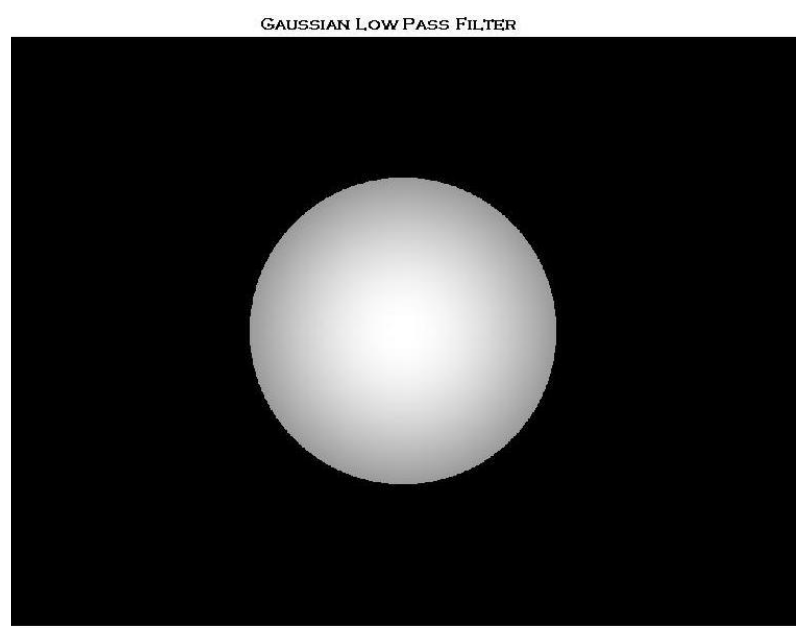

Figure 4.9 : Gaussian low pass filter (GLPF) of cutoff frequency $200, D_{0}=200$

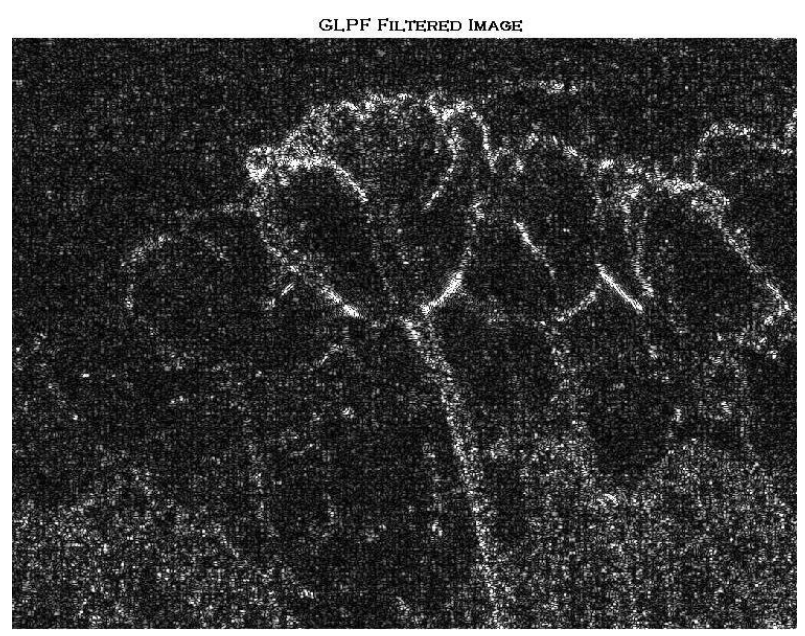

Figure 4.10: GLPF filtered image of cutoff frequency $200, D_{0}=200$

Figure 4.9 shows Gaussian low pass filter, whose circle boundary is not sharp as ideal filter, while its performance is better than lower order BLPF as shown in figure 4.10. 


\subsection{Ideal high pass filter}

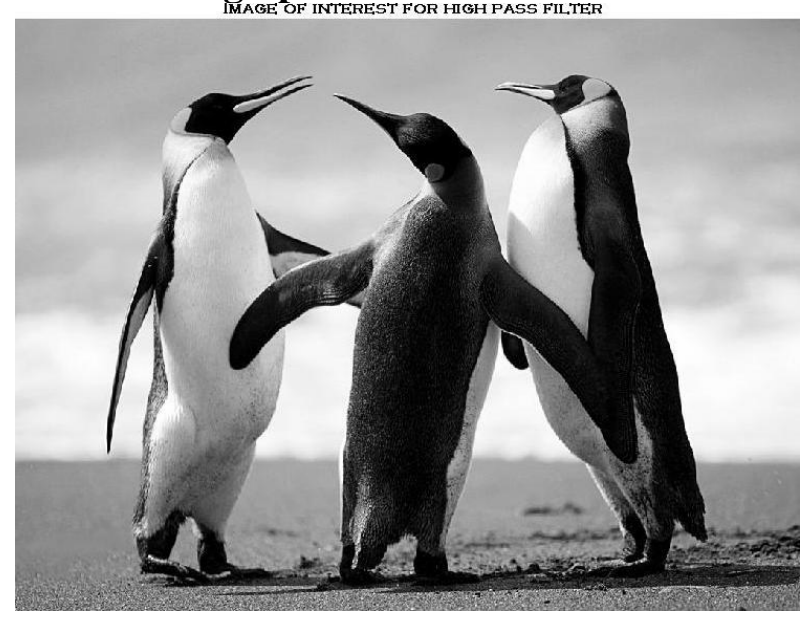

Figure 4.11: Grayscale image of interest for high pass filtering

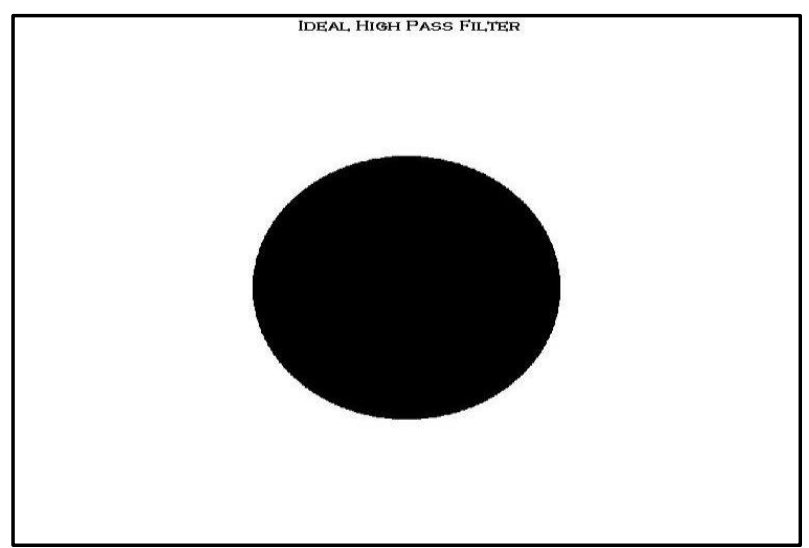

Figure 4.12: Ideal high pass filter (IHPF) of cutoff frequency $200, D_{0}=200$

For high pass filter operation we consider the image as shown in figure 4.11. Figure 4.12 shows Ideal high pass filter. Which indicate attenuation inside the circle; while outside the circle boundary represent pass-band. Figure 4.13 shows result of ideal high pass filtering. Only sharp transitions are appears in the result which is analogous to high frequency.

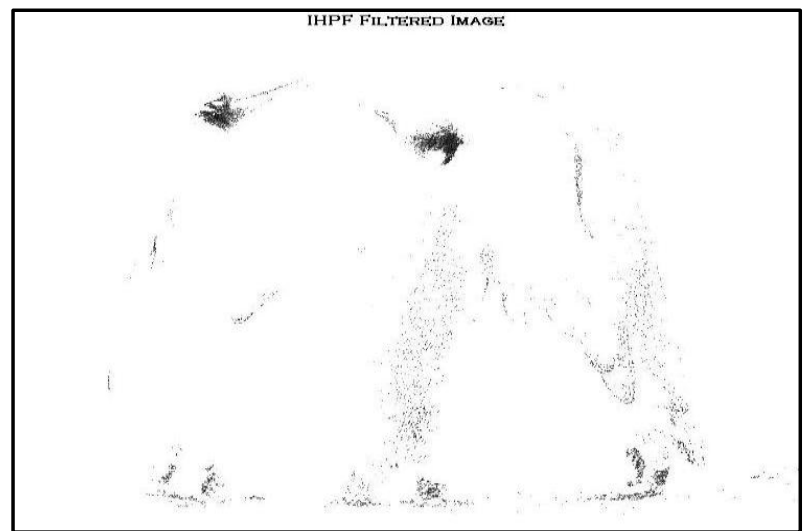

Figure 4.13: IHPF filtered image of cutoff frequency 200, $D_{0}=200$
4.5 Butterworth high pass filter

BUTTERWORTH HIGH PASS FILTER

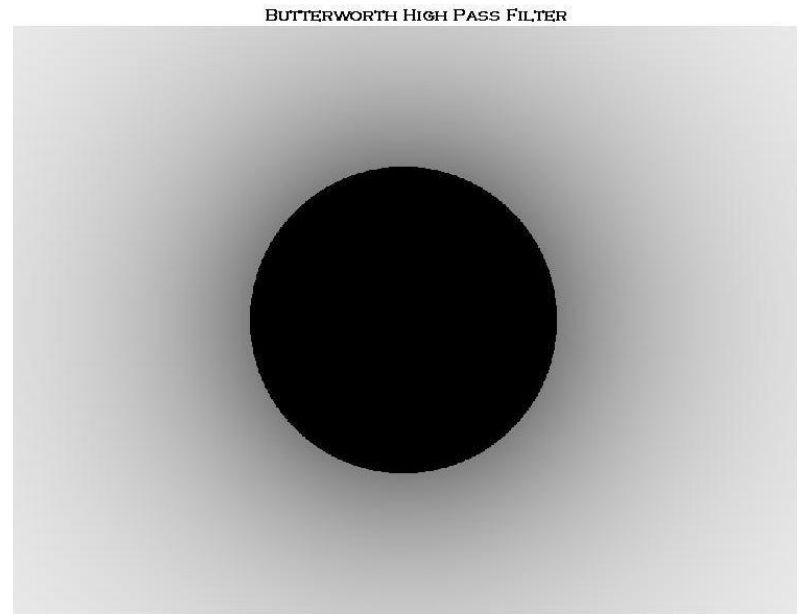

Figure 4.14 : Butterworth high pass filter (BHPF) of cutoff freauencv 200, $D_{n}=200$, order , $n=1$

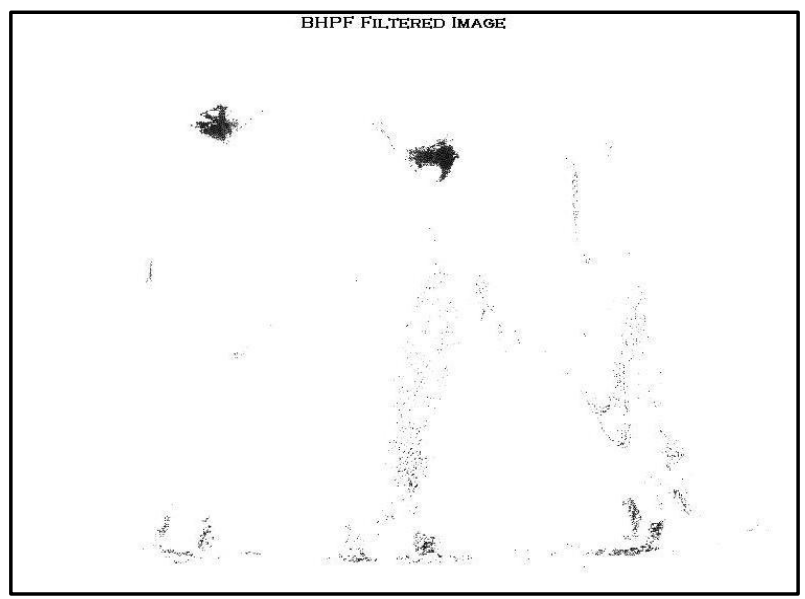

Figure 4.15: BHPF filtered image of cutoff frequency 200, $D_{0}=200$, order, $n=1$

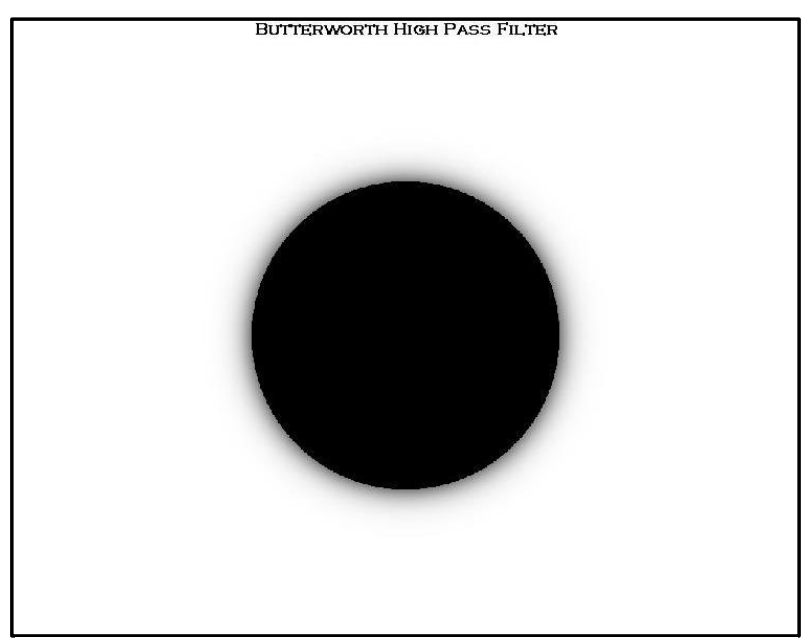

Figure 4.16 : Butterworth high pass filter (BHPF) of cutoff frequency 200, $D_{0}=200$, order,$n=10$ 


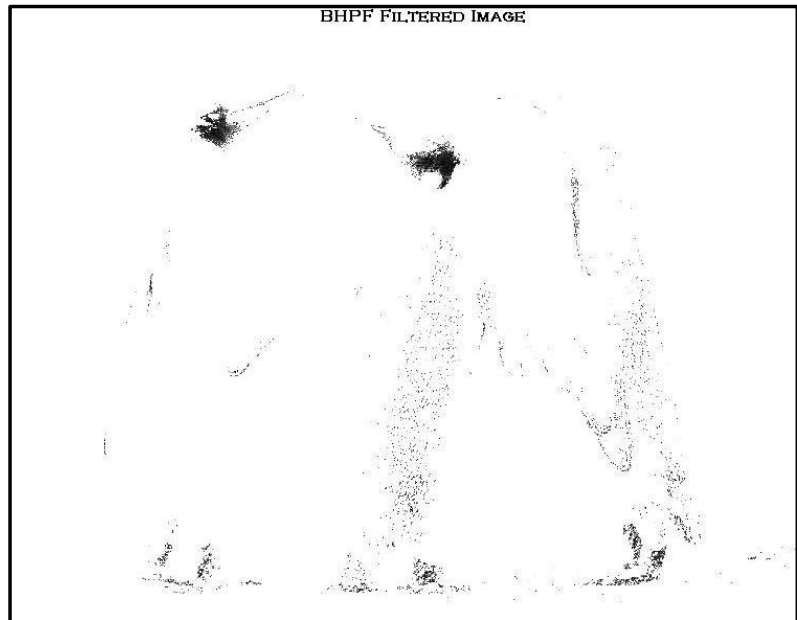

Figure 4.17 : Butterworth high pass filter (BHPF) of cutoff frequency $200, D_{0}=200$, order , $n=10$

In high pass filtering we also generate results for lower order and higher order Butterworth filter. Figure 4.14 shows first order BHPF with cutoff frequency $D_{0}=200$. Figure 4.15 is the result of this filter which is worst as the result of higher filter shown in figure 4.13. As we increases the order of BHPF as shown in figure 4.16. It approaches towards ideal filter but not exactly as it. Figure 4.17 shows the result of BHPF of order $n=10$. This result is almost similar to IHPF response.

\subsection{Gaussian high pass filter}

Gaussian high pass filter of cutoff frequency $D_{0}=200$ as shown in figure 4.18. Figure 4.19 shows the result of GHPF .It is similar to lower order Butterworth filter. However sometimes its result may be worst than lower order BHPF. It detect small high intensity transition as compared to BHPF moreover compared to IHPF. This filter taken as a generalized filter where high degree of filtering is not necessary.

To develop Ideal filter practically not possible. Higher order Butterworth filter can fulfill its requirement.

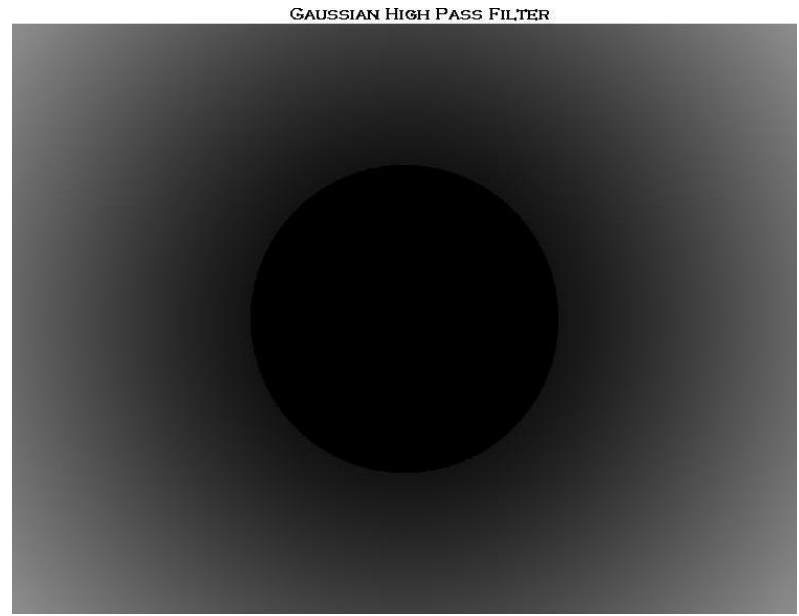

Figure 4.18: Gaussian high pass filter (GHPF) of cutoff frequency $200, D_{0}=200$

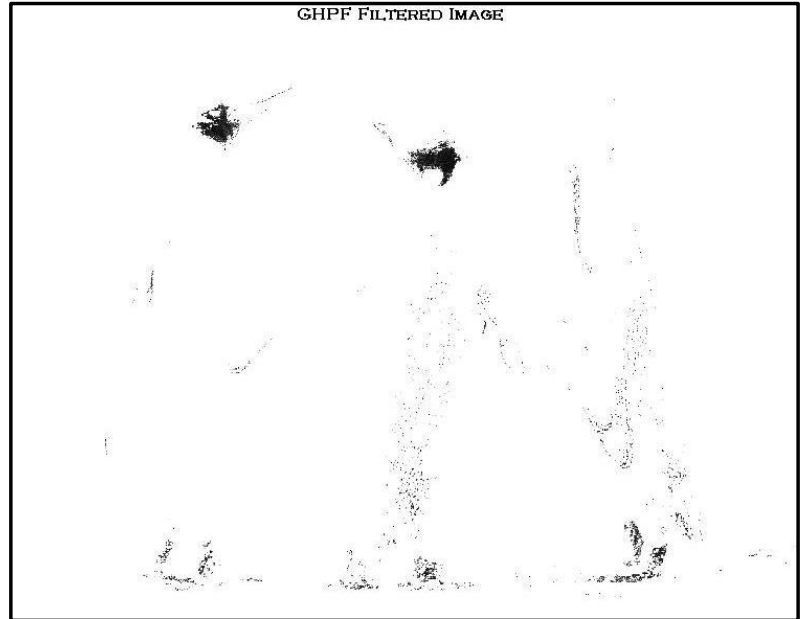

Figure 4.19: GHPF filtered image of cutoff frequency 200, $D_{0}=200$

\section{CONCLUSION}

Image enhancement is the prime aspect of digital image processing. Image filtering is an very important step in image enhancement. In our paper we implement low pass and high pass filter for same cutoff frequency. We analyze the various results as shown, after successful simulation of all these filters. Their performance depends on selection of cutoff frequency. Other than it, Ideal filter is the desire filter for both the image smoothing and sharpening. This can be achieved using higher order Butterworth filter. Especially for large images frequency domain filtering is much faster. High pass filter preserves the edges by image sharpening and shows only sharp transition of pixel intensity. While low pass filter denoises the image by smoothing the image and preserves image detail. Selection of cutoff frequency and filter order for Butterworth filter gives variable performance. Gaussian filter gives normal generalized performance exponentially.

\section{REFERENCES}

[1] Rafael C. Gonzalez, Richard E. Woods, "Digital Image Processing", Pearson Prentice Hall, Third Edition, ISBN 0-13-168728-x 978-0-13-168728-8.

[2] Sasan John jiu, "Frequency Domain Filter In Digital Image Processing In Remote Sensing: An Overview", International journal of innovative $r$ research \& development, volume2, issue 6 july, 2013, ISSN:22780211

[3] Omeed kamal khorsheed, "Produce low-pass and highpass image filter in java", International journal of Advances inengineering \&Technology, July, 2014, ISSN:22311963

[4] Snehal O.Mundhada, V.K.Shandilya, "Spatial and Transformation Domain Techniques for Enhancement", International Journal of engineering Science and Innovative technology, Volume1, Issue2, November2012 ISSN: 2319-5967.

[5] Aziz makandar, Bhagirathi Halali, "Image Enhancement Techniques using Highpass and Lowpass Filters", International journal of computer application (097588875), Volume109 No.14, January2015. 
[6] Amit shukla, Dr.R.K.Singh, "Performance analysis of frequency domain filters for noise reduction", e-Joumal of Science \& technology, (5), 9, 2014

[7] Garima Goyal, Ajay Kumar Bansal, Manish Singhal, "Review Paper on Various Filtering Techniques and Future Scope to Apply These on TEM Images" International Journal of Scientific and Research Publications, Volume 3, Issue 1, January 2013, ISSN 2250-3153

[8] Samayita Bhattacharya, Kalyani mali, "Comparative Studyof Different Filters on Images in Frequency
Domain ", International Journal of Advance Research in computer science and software Engineering, Volume4, issue8, August 2014, issue: 2277128x.s

[9] B.D.Venkatramana Reddy \& Dr.T.Jayachandra Prasad, "Frequency Domain Filtering of Colour Images using Quaternion Fourier transforms", IJCST, Vol.1, Issue2, December 2010,ISSN0976-8491.

[10] Jakia Afruz , Va'Juanna Wilson, "Frequency Domain Pseudo-color to Enhance Ultrasound Images", Canadian center of science and Education, Volume3, No.4, November 2014. 